Conclusion Adenosine-induced increase in MBF measured using perfusion mapping is accurate for the confirmation of hyperaemia during stress CMR studies and is superior to traditional, clinically used markers of adequate stress.

\section{MYOCARDIAL PERFUSION MAPPING IN CARDIAC AMYLOIDOSIS- UNEARTHING THE SPECTRUM FROM INFILTRATION TO ISCHAEMIA}

${ }^{1}$ Tushar Kotecha, 'Ana Martinez-Naharro, ${ }^{1} J a m e s$ Brown, ${ }^{1}$ Callum Little, 'Daniel Knight, ${ }^{1}$ Alexandros Steriotis, ${ }^{1}$ Niket Patel, ${ }^{1}$ Roby Rakhit, ${ }^{1}$ Philip Hawkins, 'Julian Gillmore, ${ }^{2}$ James Moon, ${ }^{3}$ Hui Xue, ${ }^{3}$ Peter Kellman, ${ }^{1}$ Marianna Fontana. ${ }^{1}$ National Amyloidosis Centre, Division of Medicine, University College London, Royal Free Hospital, London, UK; ${ }^{2}$ Barts Heart Centre, West Smithfield, London, UK; ${ }^{3}$ National Heart, Lung and Blood Institute, National Institutes of Health, Bethesda, Maryland, USA

\subsection{6/heartjnl-2019-BSCMR.19}

Background Cardiac involvement is the main driver of outcome in systemic amyloidosis, but the relationship between amyloid deposits and outcomes is not well understood. The simple explanation of physical, mechanical replacement of the interstitium by amyloid seems insufficient. Preliminary studies support the hypothesis that myocardial ischaemia could contribute to cell damage.

Purpose (1) To assess myocardial ischaemia in cardiac amyloidosis. (2) To compare patients with cardiac amyloidosis to patients assessed on invasive coronary angiography (ICA) to have normal coronary physiology (NCP), microvascular dysfunction (MVD) and triple vessel coronary disease (3VD). (3) To assess correlation of perfusion mapping to markers of disease severity and prognosis.

Methods 86 patients and 20 healthy volunteers (HV) underwent CMR at $1.5 \mathrm{~T}$ (Siemens) with standard cine, PSIR-LGE, T1,T2,Extracellular Volume (ECV) mapping and adenosine stress with myocardial blood flow (MBF) mapping. Thirtyeight patients also underwent ICA with 3 vessel assessment of Index of Microcirculatory Resistance and Fractional Flow Reserve: 7 had cardiac amyloidosis, 8 had NCP, 15 had MVD and 8 had 3 VD.

Results Cardiac amyloidosis patients had severe reduction in stress MBF and myocardial perfusion reserve (MPR) $(1.22 \mathrm{ml} /$ $\mathrm{g} / \mathrm{min} \pm 0.70$ and $1.62 \pm 0.63)$ compared to $\mathrm{HV}(3.21 \mathrm{ml} / \mathrm{g} / \mathrm{min}$ $\pm 0.64, \mathrm{p}<0.001$ and $4.17 \pm 0.78, \mathrm{p}<0.001)$, NCP $(2.66 \pm 0.56$, $\mathrm{p}<0.001$ and $2.51 \pm 0.43, \mathrm{p}=0.036)$ and MVD $(2.10 \pm 0.31$,

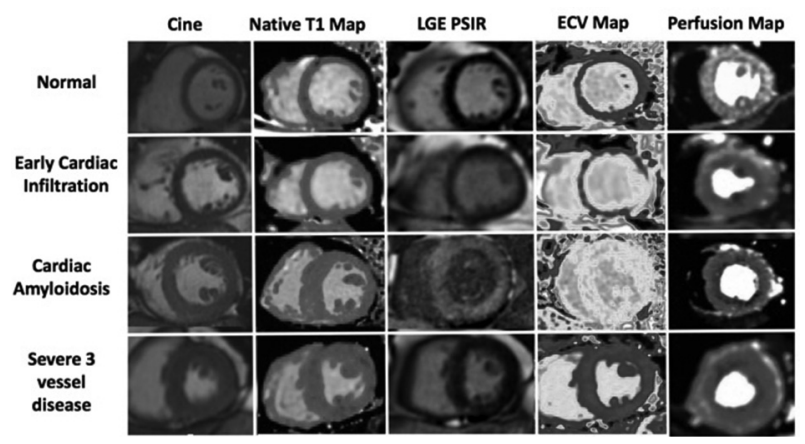

Abstract 19 Figure 1 Short axis cine SSFP images in end-diastole, corresponding native T1 mapping, late gadolinium enhancement (LGE) images, ECV Mapping and stress myocardial blood flow mapping in a normal subject, a patient with early cardiac infiltration (raised ECV, no LGE), a patient with cardiac amyloidosis, a patient with severe three vessel coronary disease $\mathrm{p}<0.001$ and $2.29 \pm 0.87, \mathrm{p}=0.014)$ with the degree of reduction being similar only to patients with $3 \mathrm{VD}(1.44 \pm 0.54$, $\mathrm{p}=1.000$ and $1.64 \pm 0.68, \mathrm{p}=1.000$ ) (figure 1). Rest $\mathrm{MBF}$ was also lower in amyloidosis than HV. Cardiac amyloidosis stress MBF and MPR inversely correlated with amyloid burden (ECV, $\mathrm{r}=-0.715, \mathrm{p}<0.001$, transmurality of LGE, $\mathrm{p}<0.01$ ), systolic dysfunction (EF, r $=0.405, \mathrm{p}<0.01)$, and blood biomarkers (NT-proBNP $(\mathrm{r}=-0.678, \mathrm{p}<0.001)$ and Troponin $\mathrm{T}$ $(\mathrm{r}=-0.628, \mathrm{p}<0.001))$. There was a correlation between stress $\mathrm{MBF}$ and native T1 $(\mathrm{r}=-0.588, \mathrm{p}<0.001)$ but not T2 $(p=0.591)$. Stress MBF and MPR were early disease markers, being elevated in patients with early cardiac amyloid infiltration (raised ECV, no LGE, $\mathrm{p}<0.01$ vsHV).

Conclusion Myocardial ischaemia is common in cardiac amyloidosis - with stress $\mathrm{MBF}$ and MPR similar to that of patients with $3 \mathrm{VD}$. The reduction correlates with the degree of amyloid infiltration and markers of adverse prognosis, highlighting the potential role of myocardial ischaemia as a key mechanism in the pathophysiology of cardiac amyloidosis.

\section{ENDOTHELIAL LOSS AS A CAUSE OF IMPAIRED MYOCARDIAL PERFUSION RESERVE ON CMR IN SEVERE AORTIC STENOSIS}

Kenneth Chan, Betty Raman, Joseph Westaby, Sairia Dass, Mario Petrou, Rana Sayeed, Houman Ashrafian, Saul G Myerson, Theodoros D. Karamitsos, Mary Sheppard, Stefan Neubauer, Masliza Mahmod. University of Oxford Centre for Clinical Magnetic Resonance Research (OCMR), Oxford, UK

\subsection{6/heartjnl-2019-BSCMR.20}

Background Impaired myocardial perfusion reserve occurs in pressure overload hypertrophy such as in severe aortic stenosis (AS) despite unobstructed epicardial coronaries. However the pathological mechanisms underlying this are poorly understood. We sought to assess myocardial perfusion reserve in severe AS by stress perfusion cardiovascular magnetic resonance (CMR), and examine the findings in relation to the histological evidence of vascular changes in the myocardium.

Methods Fourteen patients with severe AS and unobstructed epicardial coronaries underwent adenosine stress perfusion CMR before and 6 months after surgical aortic valve replacement (AVR). Myocardial biopsies were obtained during AVR and stained using CD31 +for endothelium, smooth muscle actin (SMA) for smooth muscle, and picrosirius red for

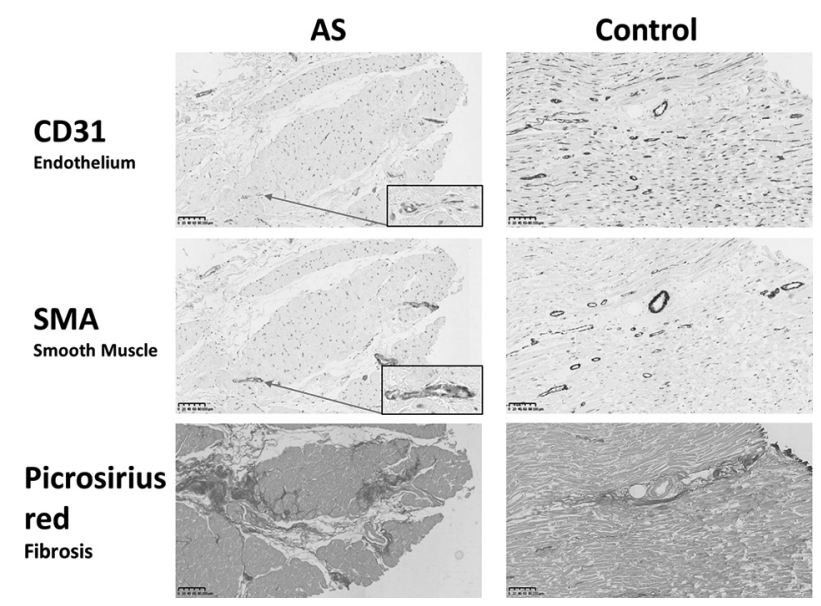

Abstract 20 Figure 1 